\title{
Electrical Stimulation of the Medial Prefrontal Cortex Increases Dopamine Release in the Striatum
}

\author{
Matthew T. Taber, B.A. and Hans C. Fibiger, Ph.D.
}

Exogenous and endogenous glutamate has been shown to evoke dopamine $(D A)$ release in the striatum using both in vitro and in vivo techniques. We hypothesized that stimulation of the prefrontal cortex (PFC) would phasically enhance striatal $D A$ release via the glutamatergic corticostriatal pathway. To test this hypothesis, in vivo brain microdialysis was employed to measure extracellular concentrations of $D A$ in the striatum during electrical stimulation of the PFC. Five rats were implanted with bilateral electrodes located in the medial PFC and dialysis probes in the dorsal striatum. Two days later the PFC of these awake, freely moving rats was stimulated first at $50 \mu A$ and then at
$100 \mu A$ for 20 minutes at 2-hour intervals. Both currents significantly increased DA release. Extracellular DA rose rapidly during stimulation, peaked immediately afterward, and then slowly returned to baseline values. Dopamine reached $118 \%$ of baseline values with $50 \mu \mathrm{A}$ stimulation and $138 \%$ with $100 \mu \mathrm{A}$ stimulation. Histologic analysis using the fluorescent retrograde dye Fluoro Gold confirmed that cells projecting to the vicinity of the striatal dialysis probe originated in the vicinity of the PFC electrodes. These results provide direct evidence for phasic, excitatory modulation of striatal DA release by the PFC. [Neuropsychopharmacology 9:271-275, 1993]
KEY WORDS: Dopamine; Electrical stimulation; Microdialysis; Prefrontal cortex; Schizophrenia; Striatum

Evidence that schizophrenia may involve both the frontal cortex and subcortical dopaminergic mechanisms has led investigators to hypothesize that this syndrome may be mediated by abnormal interactions between the prefrontal cortex (PFC) and this monaminergic system (Weinberger 1988; Robbins 1990). Cortical regulation of subcortical dopamine (DA) may be mediated through the glutamatergic corticostriatal pathway (Grace 1991; Robbins 1990). Such a role for the cortex has been supported indirectly by a number of studies. Surgical ablation of the PFC enhances stimulant effects of the amphet-

From the Division of Neurological Sciences, Department of Psychiatry, University of British Columbia, Vancouver, Canada.

Address correspondence to H.C. Fibiger, Ph.D., Division of Neurological Sciences, Department of Psychiatry, University of British Columbia, Vancouver, B.C., V6T 123 Canada.

Received May 19, 1993; revised July 21, 1993 accepted July 22, 1993. amine on locomotor behavior (Iversen 1971) and the effects of apomorphine on sterotypy-inducing properties (Scatton et al. 1982). Six-hydroxydopamine (6-OHDA) lesions of the medial PFC increase DA uptake and binding site density in the striatum (Pycock et al. 1980). Electrical stimulation of motor or visual cortex has been observed to increase striatal $\left[{ }^{3} \mathrm{H}\right] \mathrm{DA}$ release as measured by the push-pull cannula technique (Nieoullon et al. 1978). These data support a model in which cortical activity can regulate subcortical DA release.

The chemical and anatomic substrates for these observations may involve glutamatergic terminals in the vicinity of axons and terminals of nigrostriatal neurons (Grace 1991). Pharmacologic studies have found that glutamate applied to striatal slices in vitro stimulate [ $\left.{ }^{3} \mathrm{H}\right] \mathrm{DA}$ release even in the presence of tetrodotoxin (Roberts and Anderson 1979; Giorguieff et al. 1977; Marien et al. 1993). In vivo studies with push-pull cannulae have also found increased $\left[{ }^{3} \mathrm{H}\right] \mathrm{DA}$ release following local applications of low $\left(10^{-8}\right.$ to $\left.10^{-6} \mathrm{~mol} / \mathrm{L}\right)$ 
concentrations of glutamate (Cheramy et al. 1986, Leviel et al. 1990). At higher concentrations $\left(10^{-5}\right.$ to $10^{-3}$ $\mathrm{mol} / \mathrm{L}$ ), this effect was reversed, with DA release being decreased (Cheramy et al. 1986; Leviel et al. 1990). In vivo microdialysis experiments also support the facilitation of striatal DA release following a local injection of excitatory amino acids (Westerink et al. 1992, Keefe et al. 1992), although depression of DA release was not observed even at the highest concentrations used (1 mmol AMPA, $1 \mathrm{mmol} \mathrm{N}$-methyl-D-aspartate, $0.1 \mathrm{mM}$ kainate). These results show primarily, although not exclusively, facilitatory actions of glutamate on DA release in the striatum.

The relative contributions of the corticostriatal and nigrostriatal systems in regulating striatal DA release are not fully understood. Basal striatal DA release is decreased by manipulations that decrease the activity of nigrostriatal neurons, but not by local administration of glutamate receptor antagonists at concentrations that block glutamate evoked DA release (Keefe et al. 1992). These data point to a tonic role for nigrostriatal neurons in DA release with an additional phasic component being mediated by glutamatergic mechanisms.

The purpose of the present study was to investigate the role of PFC in regulating endogenous DA release in the striata of awake, freely moving animals. To this end, in vivo microdialysis was used to monitor concentrations of endogenous DA and its metabolites in rats during bilateral electrical stimulation of the PFC. Additionally, a fluorescent retrograde labeling technique was employed to confirm the origin of corticostriatal afferents innervating the region of the dialysis probe.

\section{SUBJECTS AND METHODS}

Ten male Wistar rats weighing between 320 and $360 \mathrm{~g}$ at the time of surgery were used in these experiments. The rats were anesthetized with pentobarbital (50 $\mathrm{mg} / \mathrm{kg}$ ) and were stereotaxically implanted with transverse dialysis probes passing through both striata. Coordinates were measured from bregma A: +.7; V: -4.75) according to the atlas of Paxinos and Watson (1982). The probes consisted of a hollow dialysis membrane (outer diameter $=.320 \mathrm{~mm}$; molecular weight cutoff $=60,00$; AN 69 Hospal) with an active surface length of $3.2 \mathrm{~mm}$ on each side of midline according to the lateral boundaries of each striatum. Five rats were additionally implanted with bipolar stainless-steel electrodes (.20-mm diameter; MS303/2, Plastic Products). Coordinates were measured from bregma $(\mathrm{A}:+3.0 ; \mathrm{V}$ : $3.0 ; \mathrm{L}:+.8 /-.8)$ according to the atlas of Paxinos and Watson (1982). Probe outlets and electrodes were attached to the skull via four anchoring screws and dental acrylic. Following surgery, all animals were housed individually in Plexiglas cages $(35 \times 35 \times 40 \mathrm{~cm})$ where they remained for the duration of the experiment. Subjects had free access to food and water at all times.

The five animals with electrodes and probes were used in the dialysis experiments. Forty eight hours after surgery, dialysis probes were perfused at a rate of $5 \mu \mathrm{ll}$ min with a solution containing $\mathrm{CaCl}_{2}(1.3 \mathrm{mmol} / \mathrm{L})$, $\mathrm{MgCl}_{2}(1.0 \mathrm{mmol} / \mathrm{L}), \mathrm{KCl}(3.0 \mathrm{mmol} / \mathrm{L}), \mathrm{NaCl}(147$ $\mathrm{mmol} / \mathrm{L})$, and $1 \mathrm{mM}$ phosphate buffer to maintain $\mathrm{pH}$ at 7.4. The resulting dialysate passed through outlet tubing (PE10; Clay Adams) into the sample loop of the high-performance liquid chromatography (HPLC) system. Fifty microliter samples were injected every $10 \mathrm{~min}$ utes and concentrations of DA, 3,4-dihydroxyphenylacetic acid (DOPAC), homovanillic acid (HVA), and 5-hydroxyindoleacetic acid (5-HIAA) were measured in the HPLC system using electrochemical detection (for details, see Brown and Fibiger 1992).

Prefrontal cortex stimulation began after a stable baseline was established (four samples with $<10 \%$ variation). The five rats were electrically stimulated at 50 $\mu \mathrm{A}$ (frequency $=60 \mathrm{~Hz}$, stimulus duration $=.5$ seconds, interstimulus interval $=5$ seconds) for $20 \mathrm{~min}$ utes. Two hours later all animals were stimulated again at a higher current $(100 \mu \mathrm{A}$, same parameters). These currents were chosen because rats have been found to self-administer electrical stimulation at these parameters (Phillips and Fibiger 1978) to the PFC. After another 2-hour period, the rats were removed from their cages and sacrificed; brains were cut in $50 \mu \mathrm{mol}$ sections and stained with cresyl violet to confirm electrode and probe placements.

Retrograde labeling of cells innervating the region of the dialysis probe was performed on the remaining five rats using fluorescence histology. Striatal probes

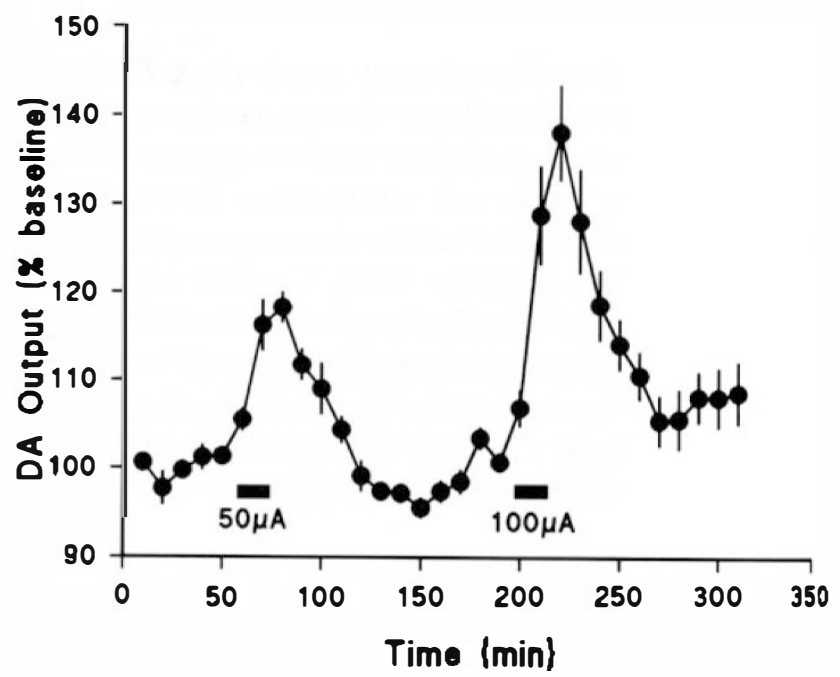

Figure 1. Effects of electrical stimulation of the PFC at 50 and $100 \mu \mathrm{A}(60 \mathrm{~Hz}$, pulse duration $=.5$ seconds, interstimulus interval $=5$ seconds) on dialysate concentrations of DA in the striatum $(n=5)$. 


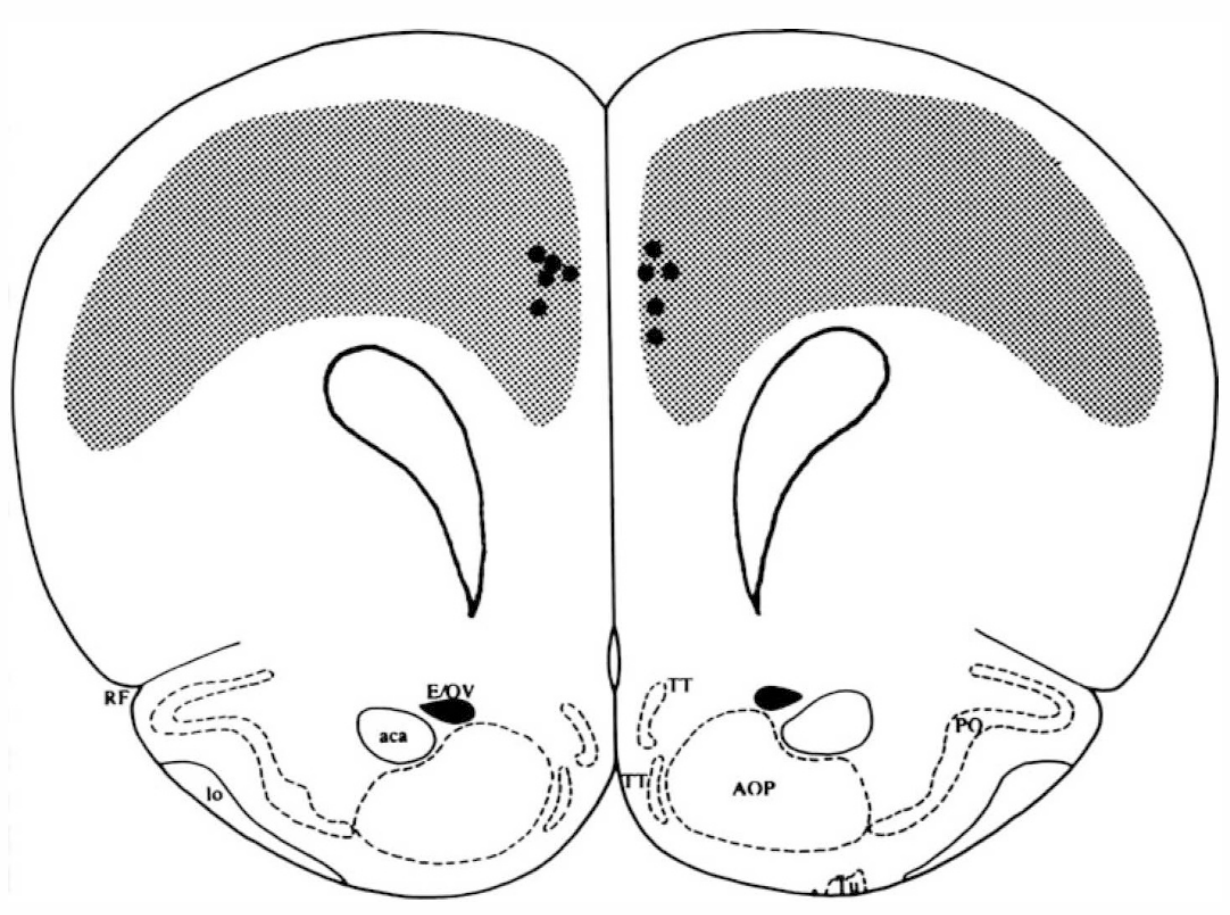

Figure 2. Composite diagram of afrontal section showing the electrode tip placements in five rats (solid circles) and the area containing the densest population of FG labeled cells (stippled)following Fluoro Gold infusion into the striatal dialysis probe. Adapted from the atlas of Paxinos and Watson (1982). Bregma $+3.2 \mathrm{~mm}$. were filled with a solution containing $4 \%$ Fluoro Gold in saline $(.9 \%)$ and were flushed with saline 18 hours later. Five days later these animals were given a lethal injection of pentobarbital and were perfused with $4 \%$ paraformaldehyde. Following a 2-hour postfix, brains were bathed overnight in $.05 \mathrm{~mol} / \mathrm{L}$ phosphate buffer, cut in $35 \mu \mathrm{m}$ coronal slices, and mounted on slides. The sections were observed under a fluorescent microscope using ultraviolet light at a wavelength of 340 to $380 \mathrm{nmol}$.

To analyze the microdialysis results, the average of the first four samples with $<10 \%$ variability was considered to make up baseline, and was defined as $100 \%$. Data were analyzed using one-way and two-way analysis of variance (ANOVAs) with repeated measures.

\section{RESULTS}

The average basal extracellular DA output was $15.0 \pm$ $2.2 \mathrm{fmol} / \mathrm{min}$. Electrical stimulation increased DA output in all animals, and the increase was found to rise with current intensity (Fig. 1). Fifty microAmperes increased DA output to a maximum of $118 \%$ of baseline values; $100 \mu \mathrm{A}$ increased DA output to a maximum of $138 \%$ of baseline values. The effects of both stimulation currents were significant across time $(p<.001)$. A significant stimulation intensity-by-time interaction was also found across the two levels of stimulation $(p<.02)$. The DA metabolites DOPAC and HVA and the serotonin metabolite 5-HIAA were also increased by both levels of stimulation $(p<.001$; data not shown), although in all cases the peak percent increase was lower than the corresponding DA values. The DOPAC levels peaked at $117 \%$ and $128 \%$ for $50 \mu \mathrm{A}$ and $100 \mu \mathrm{A}$, respectively, HVA peaked at 113 and $121 \%$, and 5-HIAA peaked at $106 \%$ and $107 \%$. Significant stimulation intensity-by-time interactions were found for DOPAC $(p<.002)$ but not HVA or 5-HIAA.

Dopamine concentrations rose rapidly during each 20-minute stimulation and peaked in the 10-minute sample immediately following the end of the stimulation period. Thereafter, DA decreased and returned to baseline 50 to 60 minutes after PFC stimulation. Formal behavioral measures were not performed, but casual observation indicated that the stimulation did not produce behavioral activation. Indeed, rats appeared more active during the $50-\mu \mathrm{A}$ stimulation than at $100 \mu \mathrm{A}$.

Histologic analysis of the five rats with electrodes and dialysis probes implanted confirmed all placements. Electrodes were located in medial PFC at $3.0 \mathrm{~mm}$ to $3.2 \mathrm{~mm}$ anterior to bregma, $2.5 \mathrm{~mm}$ to $3.0 \mathrm{~mm}$ ventral to skull surface, and within $1 \mathrm{~mm}$ of the midline (Fig. 2). All dialysis probes were located in dorsal striatum $.5 \mathrm{~mm}$ to $.7 \mathrm{~mm}$ anterior to bregma.

In rats infused with Fluoro Gold, fluorescence was examined in coronal slices from the frontal pole to the dialysis probe. The area of densest retrograde labeling was the dorsal cortex in all sections viewed, and the labeling extended across the entire medial/lateral aspect of this region. Dense labeling was found in the area surrounding the electrode sites in all animals viewed. These data indicate that the medial PFC in the vicinity of the stimulation electrodes project directly to the region of the striatum containing the dialysis probe. This 
confirms earlier retrograde (McGeorge and Faull 1989) and anterograde (Berendse et al. 1992) labeling studies.

\section{DISCUSSION}

The primary result presented here is direct evidence that increased activity in the medial PFC can evoke subcortical DA release. The stimulation parameters that were used ( 50 and $100 \mu \mathrm{A}$ ) have been found to maintain self-stimulation in rats (Phillips and Fibiger, 1978) and can therefore be considered behaviorally relevant. No behavioral abnormalities such as seizures were observed in any animal. The time course of the increased DA output is most consistent with a phasic excitatory regulation of DA release. During stimulation, DA concentrations rose rapidly, peaked immediately after cessation of the stimulation, and then declined. Additionally, preliminary experiments (Taber and Fibiger, unpublished results) indicate that equivalent electrical stimulation of the PFC elevates striatal acetylcholine release. These results demonstrate the ability of the microdialysis technique to detect transient neurotransmitter fluctuations following discrete physiologic, phasic events.

Nieoullon et al. (1978) have reported that cortical stimulation produces long-lasting increases in extracellular concentrations of $\left[{ }^{3} \mathrm{H}\right] \mathrm{DA}$ in the striatum. Specifically, extracellular DA concentrations were still rising an hour after termination of the cortical stimulation. This result led to the hypothesis that the corticostriatal projection has a tonic facilitory influence on striatal DA release (Nieullon et al. 1978; Romo et al. 1986; Grace 1991). However, later studies have indicated that glutamate in the striatum does not have a tonic influence on DA release (Keefe et al. 1992; Leviel et al. 1990) and suggest that exogenous glutamate produces phasic increases in striatal DA release. The present results are consistent with this view in that they demonstrate that PFC stimulation produces transient increases in DA release, an effect that may be mediated by the glutamatergic corticostriatal projection.

It is unclear why the DA increases seen in the study by Nieoullon et al. (1978) lasted so much longer than those in the current study. Technical differences suggest three possibilities. First, the cats in the Nieoullon study were stimulated at a much higher frequency (300 $\mathrm{Hz}$ ) and in a different area of the cortex (motor) than in the current study. The second involves behavioral state; the rats in the current experiment were awake and not restrained, whereas the cats in the Nieoullon study were anesthetized. Keefe et al. (1992) suggests that behavioral state may contribute to baseline levels of DA mediated through the corticostriatal projection; perhaps behavioral state also affects stimulation evoked
DA release. The third possibility is that the dialysis and push-pull cannula techniques are measuring different pools of DA. Microdialysis samples total extracellular DA concentrations, whereas the push-pull cannula technique used by Nieoullon et al. measured recently synthesized $\left[{ }^{3} \mathrm{H}\right] \mathrm{DA}$. It is possible that cortical stimulation influences release of [ $\left.{ }^{3} \mathrm{H}\right] \mathrm{DA}$ differently than it affects endogenous stores of DA.

The present data stand in contrast to earlier lesionbased studies that have suggested that the prefrontal cortex has inhibitory actions on subcortical dopaminergic systems (Iversen 1971; Pycock et al. 1980; Jaskiw et al. 1990). The chronic and irreversible nature of PFC lesions used in those studies makes direct comparisons with the present acute manipulations difficult. Clearly, however, the present findings point to an excitatory influence of the PFC on subcortical DA systems in awake, relatively intact, behaving animals. A report from Louilot et al. (1989) is somewhat more difficult to reconcile with the present results. These authors found that infusions of tetrodotoxin into the PFC increased extracellular concentrations of DOPAC in the nucleus accumbens, suggesting that the PFC normally inhibits DA metabolism in this structure. In the present experiments, electrical stimulation of the PFC increased extracellular concentrations of DOPAC in the dorsal striatum. The basis of these apparent discrepancies is not presently known and requires investigation.

Anatomic systems other than the glutamatergic corticostriatal projection may have contributed to the stimulation-induced increase in DA release observed in the present experiments. Most significantly, efferents from the PFC have recently been found to synapse directly on tyrosine hydroxylase-containing neurons of the ventral tegmental area (Sesack and Pickel 1992). A projection from the PFC also terminates in the substantia nigra (Gerfen et al. 1982). Excitation of either of these systems may have activated dopaminergic projections to the striatum. Another channel through which stimulation of the PFC could evoke striatal DA release involves the intralaminar nuclei of the thalamus. For example, the centrolateral nucleus sends axon collaterals to both the cortex and the striatum (Cesaro et al. 1979). Antidromic stimulation of the projection to the PFC may have activated striatal collaterals to evoke DA release. Experiments are currently in progress to determine the neural systems through which stimulation of the PFC enhances DA release in the striatum.

\section{ACKNOWLEDGMENTS}

This work was supported by the Medical Research Council of Canada (PG-23) and an Unrestricted Grant from BristolMyers Squibb. The excellent technical assistance of Catriona Wilson is gratefully acknowledged. 


\section{REFERENCES}

Berendse HW, deGraaf YG, Groenewegen HJ (1992): Topographical organization and relationship with ventral striatal compartments of prefrontal corticostriatal projections in the rat. J Comp Neurol 36:314-347

Brown EE, Fibiger HC (1992): Cocaine induced conditioned locomotion: Absence of associated increases in dopamine release. Neuroscience 48:621-629

Cesaro P, Nguyen-Legros J, Berger B, Alvarez C, Albe-Fessard D (1979): Double labelling of branched neurons in the central nervous system of the rat by retrograde axonal transport of horseradish peroxidase and iron dextran complex. Neurosci Lett 15:1-7

Cheramy A, Romo R, Godheu G, Baruch P, Glowinski J (1986): In vivo presynaptic control of dopamine release in the cat caudate nucleus. II. Facilitatory or inhibitory influence of L-glutamate. Neuroscience 19:1081-1090

Gerfen CR, Staines WA, Arbuthnot GW, Fibiger HC (1982): Crossed connections of the substantia nigra in the cat. J Comp Neurol 207:283-303

Grace AA (1991): Phasic versus tonic dopamine release and modulation of dopamine system responsivity: A hypothesis for the etiology of schizophrenia. Neuroscience 41:1-24

Giorguieff MF, Kemel ML, Glowinski J (1977): Presynaptic effect of L-glutamic acid on the release of dopamine in striatal slices. Neurosci Lett 6:73-77

Iversen SD (1971): The effect of surgical lesions to frontal cortex and substantia nigra on amphetamine responses in rats. Brain Res 31:295-311

Jaskiw GE, Karoum F, Freed WJ, Phillips I, Kleinman JE, Weinberger DR (1990): Effect of ibotenic acid lesions of the medial prefrontal cortex on amphetamine-induced locomotion and regional brain catecholamine concentrations in the rat. Brain Res 534:263-272

Keefe KA, Zigmond MJ, Abercrombie ED (1992): Extracellular dopamine in striatum: Influence of nerve impulse activity in medial forebrain bundle and local glutamatergic input. Neuroscience 47:325-332

Leviel V, Gobert A, Guibert B (1990): The glutamate mediated release of dopamine in the rat striatum: further characterization of the dual excitatory-inhibitory function. Neuroscience 39:305-312

Louilot A, Le Moal M, Simon H (1989): Opposite influences of dopaminergic pathways to the prefrontal cortex or the septum on the dopaminergic transmission in the nucleus accumbens. An in vivo voltammetric study. Neuroscience 29:45-56
Marien M, Brien J, Jhamandas K (1983): Regional release of $\left[{ }^{3} \mathrm{H}\right]$ dopamine from rat brain in vitro: Effects of opioids on release induced by potassium, nicotine, and L-glutamic acid. Can J Physiol Pharmacol 61:43-60

McGeer PL, McGeer EG, Scherer U, Singh K (1977): A glutamatergic corticostriatal path? Brain Res 128:369-373

McGeorge AJ, Faull RL (1989): The organization of the projection from the cerebral cortex to the striatum in the rat. Neuroscience 29:503-537

Nieoullon A, Cheramy A, Glowinski J (1978): Release of dopamine evoked by electrical stimulation of the motor and visual areas of the cerebral cortex in both caudate and in the substantia nigra in the cat. Brain Res 145:69-83

Paxinos G, Watson C (1982): The Rat Brain in Stereotaxic Coordinates. New York, Academic Press

Phillips AG, Fibiger HC (1978): The role of dopamine in maintaining intracranial self-stimulation in the ventral tegmentum, nucleus accumbens, and medial prefrontal cortex. Can J Physiol 32:58-66

Pycock CJ, Kerwin RW, Carter CJ, (1980): Effect of lesions of cortical dopamine terminals on subcortical dopamine in rats. Nature 286:74-77

Robbins TW (1990): The case for frontostriatal dysfunction in schizophrenia. Schizophr Bull 16:391-402

Roberts PJ, Anderson SD (1979): Stimulatory effect of L-glutamate and related amino acids on $\left[{ }^{3} \mathrm{H}\right]$ dopamine release from rat striatum: An in vitro model for glutamate actions. J Neurochem 32:1539-1545

Romo R, Cheramy A, Godeheu G, Glowinski J(1986): In vivo presynaptic control of dopamine release in the cat caudate nucleus. III. Further evidence for the implication of corticostriatal neurons. Neuroscience 19:1091-1099

Scatton B, Worms P, Lloyd KG, Bartholini G (1982): Cortical modulation of striatal function. Brain Res 232:331-343

Sesack SR, Pickel VM (1992): Prefrontal cortical efferents in the rat synapse on unlabeled neuronal targets of catecholamine terminals in the nucleus accumbens septi and on dopamine neurons in the ventral tegmental area. J Comp Neurol 320:145-160

Weinberger DR, Berman KF, Illowsky BP (1988): Physiological dysfunction of dorsolateral prefrontal cortex in schizophrenia. iii. A new cohort and evidence for a monoaminergic mechanism. Arch Gen Psychiatry 49:609-615

Westerink BHC, Santiago M, De Vries JB (1992): The release of dopamine from nerve terminals and dendrites of nigrostriatal neurons induced by excitatory amino acids in the conscious rat. Naunym Schmeiderbergs Arch Pharmacol 345:523-529 